\title{
Caudal Regression Syndrome - A Case Report and Literature Review
}

\author{
S BEGUM ${ }^{\mathrm{a}}$, R SHELIM ${ }^{\mathrm{b}}$, T BEGUM ${ }^{\mathrm{c}}$, N NAHAR ${ }^{\mathrm{d}}$
}

\begin{abstract}
:
Background and Purpose: Caudal regression syndrome (CRS) is a rare congenital abnormality in which a segment of the spine and spinal cord fails to develop. The severity of the morphologic derangement inversely correlates with residual spinal cord function. The caudal regression syndrome is frequently associated with maternal diabetes. The exact etiology is elusive, though maternal diabetes is one of the important factor; genetic factors, and hypoperfusion might play roles. Recently, the role of teratogens has been studied in animal. Here we report a case of CRS of a newborn baby of diabetic mother.
\end{abstract}

\section{Introduction:}

Incidence of CRS varies from 1:7500 to 1:60,000 live births ${ }^{1}$. The majority of cases of CRS are sporadic, partial genetic contribution has been reported ${ }^{2}$. However it occurs in about one in 350 infants of diabetic mothers, which is about 200-fold increase incidence in diabetic patient than general population ${ }^{3}$.

The syndrome has been occurring more frequently in the offspring of diabetic than non-diabetic mothers. Although hyperglycemia in the early stages has been implicated, the pathogenesis remains unknown. Trauma, nutritional problems, toxic agents and genetics are the other factors suggested in the aetiology 4 .

It has also been hypothesized that hyperglycemia leads to release of free radicals from the influx of glucose across injured cell and mitochondrial membranes. These excess free radicals can be teratogenic; prostaglandin

a. Dr. Suraiya Begum, FCPS, Registrar, Department of Paediatrics, BIRDEM and Ibrahim Medical College

b. Dr Rumana Shelim, MBBS, Medical Officer, Department of Paediatrics, BIRDEM and Ibrahim Medical College

c. Prof. Tahmina Begum, FCPS, M. MED, Professor and Head of the Depatment of paediatrics, BIRDEM and Ibrahim Medical College

d. Prof. Nazmun Nahar, FCPS, FRCP, Professor of Paediatrics, BIRDEM and Ibrahim Medical College

Address of correspondence: Dr. Suraiya Begum, FCPS, Email: suraiyadr07@yahoo.com

Received: 11 January, 2010

Accepted: 29 December, 2010
Methods: The history of the patient was taken from parents and physical examination was done. Plain radiographs, USG of abdomen and other investigations were done for evaluation.

Results: Agenesis of lower three thoracic, lumbar and sacral vertebrae with multiple congenital anomalies were observed. Lower limbs showed hypoplastic and talipes equinovarus. Hip joints were fixed, flexion contracture of the knees and webbing of Popliteal fossa were present. Other anomalies were dextrocardia and duplex right kidney.

Conclusion: CRA is a rare congenital anomaly associated with maternal diabetes. Control of diabetes is necessary to reduce the risk of occurrence.

(J Bangladesh Coll Phys Surg 2011; 29: 166-169)

imbalance, amino acid abnormalities ultimately leading to disruption in signal transduction ${ }^{5}$. Animal experiments have shown that CRS could be induced by retinoic acid, diethylpropion, lithium, sulfamide, cadmium, lead, ochratoxin A, radiation, hyperthermia, organic fat solvents, and 6- aminonicotinamide. In addition vitamin A deficiency may be responsible for CRS. Familial occurrence of CRS has been reported, but no Mendelian pattern of inheritance has been established $^{2}$. A recent study provides evidence of the homeobox gene HLXB9, but a possible role for this gene has not been established yet ${ }^{6}$.

CRS is thought to arise from a defect in induction of caudal elements of the embryo before the 7th week of gestation ${ }^{7}$. The exact process leading to the development of CRS has not been established, although it has been proposed that one or more processes of primitive streak migration, primary or secondary neurulation, or differentiation are compromised ${ }^{4,5}$. Bohring et al. ${ }^{8}$ postulate that the spectrum of congenital malformations observed in CRS represents abnormalities in blastogenesis and is due to disturbances of a primary embryonic field. The defects of the primary field lead to failure of axis formation, midline, primitive node and streak, gastrulation, segmentation of the paraxial mesoderm, laterality determination and cardiac formation. 
CRS is characterized by agenesis of spine to a variable degree. The orthopedic, gastrointestinal, genitourinary and cardiac anomalies are commonly seen with this condition $^{9}$. CRS may range from absent coccyx as an isolated finding without neurologic sequelae to sacral or lumbosacral agenesis ${ }^{10}$. Akhter et al. have described a syndrome of sirenomelia in which the baby had limb fusion, tracheoesophageal fistula and absent genitalia ${ }^{11}$. In less complete varieties there may be only sacral hypoplasia and urinary tract infection; therefore it has been suggested that the sacrum should be examined in all cases of urinary tract infections in the child born to diabetic mothers ${ }^{12}$.

Diagnosis of CRS can be made in the first trimester by noting the short crown - rump length. Sonography in second or third trimester can also demonstrate the absence of sacrum and shortened femurs. The legs may be flexed and abducted at the hips, and there may be clubfeet. Sonography may detect associated urinary anomalies, such as renal agenesis, cystic dysplasias, caliectasis and gastro-intestinal anomalies, such as duodenal atresia. Diagnosis is usually made on routine prenatal ultrasound examination. In cases with oligohydramnios in whom diagnosis is not clear, an MRI examination may be performed ${ }^{13,14}$. We are reporting this case because of rarity.

\section{Case report:}

A 30 year-old diabetic mother gave birth to a female baby following 37 weeks gestation by caesarean section . Indication of caesarean section were gestational Diabetes mellitus, pregnancy induced hypertension and breech presentation.

Maternal diabetes was detected at third trimester. She was on irregular antenatal check up. Her $\mathrm{HbA}_{1} \mathrm{C}$ was 9.1\%. Antenatal ultrasound done on single occasion showed oligohydromnious but no fetal abnormalities were detected.

At birth, we noted multiple abnormalities in the baby. Her weight was 2511g (AGA), length $40 \mathrm{~cm}$ (below third centile). Head circumference was $35 \mathrm{~cm}$ (AGA) but dolicocephalic with normal anterior fontanelle. She had low hair line and low set ear. Chest examination showed broad chest with wide apart nipples, apex beat was on right 4th intercostal space, heart sounds were more prominent on right side and no murmur was present. Lower limbs showed hypoplasia muscle wasting and talipes equinovarus. Lower thoracic, lumbar and sacral vertebrae could not be palpated. Hip was also hypoplastic and there were dimples on the lateral aspect of both thighs overlying the greater trochanters. Hip joints were fixed in position of flexion, abduction and external rotation. There was flexion contracture of the knees and webbing of Popliteal fossa. Movement of joints were restricted in lower limbs. The bladder was not distended and the anus was normal in position. Her upper limbs were normal. XRay of skeleton showed 8th \& 9th thoracic hemi vertebrae, agenesis of lower three thoracic, lumbar and sacral vertebrae, anterior widening of all ribs, anterior forking of right 6th \& 8th ribs, rudimentary hip bones, non ossification of left fibula and fracture of left femur and tibia. were noted. Chest x-ray showed dextrocardia, USG of abdomen revealed duplex right kidney.

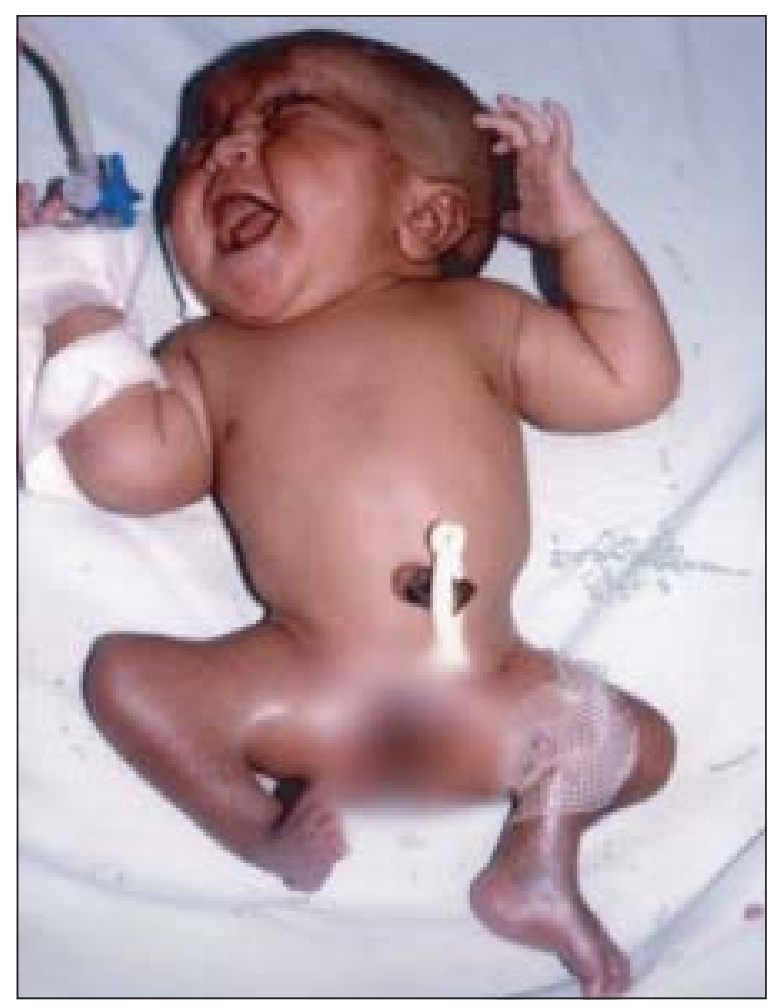

Fig-1: Shows flexion contracture of the knees, webbing of Popliteal fossa and talipes equinovarus.
Fig-2: rudim 


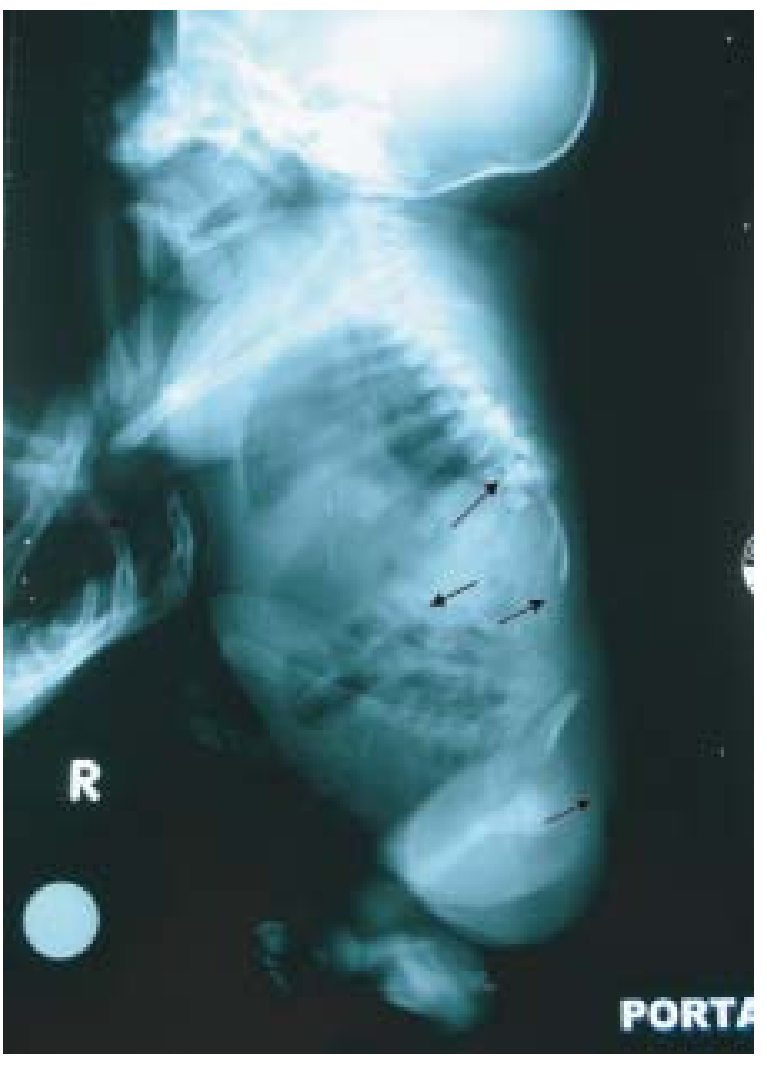

Fig-2: Shows 8th \& 9th thoracic hemi vertebrae, agenesis of lower three thoracic, lumbar and sacral vertebrae and rudimentary hip bones.

\section{Discussion:}

CRS occurs in 0.2 to $1 \%$ of pregnancies of women with diabetes, and about $15-25 \%$ of cases of CRS are associated with either type I or type II diabetes mellitus in the mother ${ }^{15}$. Women with diabetes who are dependant on insulin are 200-400 times more likely to have a child with CRS than women without diabetes. Thus making CRS the most characteristic foetal abnormality of diabetic embryopathy ${ }^{16}$.

In patients with good diabetic control during the first few weeks of pregnancy, the risk of congenital malformations is not significantly different from that of the general population. However, history of maternal diabetes is present in only $16 \%$ to $22 \%$ of infants with CRS indicating an environmental contributing factor ${ }^{17}$. In our case CRS was probably due to maternal uncontrolled diabetes; although diabetes was diagnosed in third trimester but we can not be very sure about the glycaemic control in first trimester as the glucose profile and $\mathrm{HbA}_{1} \mathrm{C}$ could not be done as needed due to the poor compliance of the patient.

Depending upon the extent of spinal malformation, five types have been described by Renshaw, the most severe being sirenomelia ${ }^{18}$. According to Renshaw the patient in this study can be classified as Type III.

Orthopaedic anomalies like gluteal anomalies, scoliosis, and talipes deformities in 12\%, and progressive deficits like back and leg pain present in 30\% cases in different study $^{7}$, But in our patient lower limbs was hypoplastic with muscle wasting and talipes equinovarus. There was also fixed flexion, abduction and external rotation of hip joints; webbing of popliteal fossa and restricted movement of joints of lower limbs in our patient. In one study, hip-knee-flexion contracture, equinovarus deformity of foot associated with popliteal webbing were the prominent orthopaedic abnormalities, there was no associated upper limb abnormalities which was similar to our study ${ }^{19}$. In same study radiographic documentation showed total agenesis of the sacrum with subtotal lumbar agenesis and the lowest lumbar vertebrae were resting above an iliac amphiathrosis ${ }^{19}$; in the present study lower thoracic, lumbar and sacral vertebrae could not be shown radiologically. In another study, a baby girl was delivered whose lower limbs were hypoplastic and held in a position of flexion both at the hips as well as the knees. She had bilateral talipes equinovarus ${ }^{20}$.

Congenital cardiac disease was found in $24 \%$ cases with CRS in which Tetralogy of Fallot is the most common cardiac anomaly ${ }^{1}$. Martinez-Frias presented data suggesting that the incidence of transposition of the great vessels was significantly increased in the offspring of diabetic mothers ${ }^{21}$. Dextrocardia was detected on postnatal examination in present study that was different from other study.

Other malformations such as Chiari I malformation is associated with caudal regression syndrome ${ }^{22}$. In the present case, no cerebral anatomical abnormality was seen. Genito-urinary disease like hydronephrosis, renal agenesis, epispadias and hypospadias was present in $24 \%$ cases, but in our patient duplex kidney was detected by ultrosonography ${ }^{4,5}$.

The prognosis depends on the extent of spinal abnormalities. The greater the extent of spinal anomalies, the greater the neurological deficits and 
consequently the greater orthopaedic and neurological support is required. The main cause of morbidity is usually neurogenic bladder causing urologic impairment and progressive renal failure ${ }^{4}$. The prognosis in this neonate was unlikely to be good as there was high termination of spine and fixed flexion of both the lower limbs; long-term neurological, urologic and orthopaedic complications may develop.

\section{Conclusions:}

It has been known that diabetes in pregnancy can have severe adverse effects on foetal and neonatal outcomes. CRA is an uncommon abnormality, the associated disabilities can be severe and genetic counselling is necessary. In this case maternal diabetes may be inciting agent. Treatment is difficult and multidisciplinary, largely supportive, need intensive and long-term attention, so prevention the ultimate goal. Control of diabetes before conception and in early pregnancy is presumed to reduce the risk of occurrence.

\section{References:}

1. Singh SK, Singh RD, Sharma A. Caudal regression syndrome: A case report and review of literature. Pediatr Surg Int 2005; 21:578-81.

2. Zaw W, Stone DG. Caudal regression syndrome in twin pregnancy with type II diabetes. J Perinatol 2002; 22: 17174.

3. Sen KK, M Patel. Caudal regression syndrome MJAFI 2007; 63: $178-79$

4. Towfighi J, Housman C. Spinal cord abnormalities in caudal regression syndrome. Acta Neuropathol (Berl). 1991; 81:45866.

5. Reece EA, Homko CJ. Why do diabetic women deliver malformed infants? Clin Obstet Gynecol 2000; 43:32-45.

6. Ross AJ, Ruiz - Perez V, Wang Y, et al. A homeobox gene, HLXB9, is the major locus for dominantly inherited sacral agenesis. Nat Genet 1998; 20:358-61.

7. Sadler TW. Langman's Medical Embryology. 8th ed. Philadelphia, Pa: Lippincott Williams \& Wilkins, 2000; 61110.

8. Bohring A, Lewin OS, Reynolds FJ, et al. Polytopic anomalies with agenesis of the lower vertebral column. Am J Med Genet 1999; 87:99-114.
9. Tortori-Donati P, Fondelli MP, Rossi A, Charles AR, Armando C, Valeria C. Segmental spinal dysgenesis: neuro-radiologic findings with clinical and embryologic correlation. Am J Neuroradiol 1999; 20: 445-56.

10. Stocker JT, Heifetz SA. Sirenomelia: A morphological study of 33 cases and review of the literature. Perspect Pediatr Pathol 1987; 10:7.

11. Anne G Osborn. Diagnostic Neuropathology. Normal anatomy and congenital anomalies of the spine and spinal cord. 1st ed. Missouri: Mosby Inc, 1994; 19:809-10.

12. Akhtar J, Shaikh NA, Taqvi SR, et al. Sirenomelia (mermaid baby). J Coll Physicians Surg Pak 2005; 15:736-7.

13. Baxi L, Warren W, Collins MH, et al. Early detection of caudal regression syndrome with transvaginal scanning. Obstet Gynecol 1990; 75:486.

14. Carol M R, Stephanie R Wilson, William Charboneau, JoAnn M Johnson. Diagnostic ultrasound. In: Eric E. Sauerberi, ed. The Fetal Spine. 3rd ed. Missouri: Mosby Inc, 2005; 38:1296.

15. Towfighi J, Housman C. Spinal cord abnormalities in caudal regression syndrome. Acta Neuropathol. (Berl). 1991; 81:45866.

16. Adra A, Cordero D, Mejides A, Yasin S, Salman F, O’Sullivan MJ. Caudal regression syndrome: etiopathogenesis, prenatal diagnosis and perinatal management. Obstet. Gynecol Surg. 1994; 49: 508-16.

17. Passarge E, and Lenz W. Syndrome of caudal regression in infants of diabetic mothers: Observations of further cases. Pediatrics 1966; 37:672-5.

18. Renshaw TS. Sacral Agenesis. The Pediatric Spine - Principles and Practice. New York: Raven Press, 1994; 1:2214.

19. Ali Al Kaissi, Klaus Klaushofer Franz Grill. Caudal regression syndrome and popliteal webbing in connection with maternal diabetes mellitus: A case report and literature review. Cases J. 2008; 1:407.doi: 10.1186/1757-1626-1- 407.

20. Fayyaz A, Ilyas M and Iqbal O. Prenatal diagnosis of caudal regression syndrome Journal of College of Physians \& surgeons, Pakistan 2007; 17:425-6.

21. Martinez-Frias M, Bermejo E, Rodriguez-Pinilla E, Prieto L, Frias JL: Epidemiological analysis of outcomes of pregnancy in gestational diabetic mothers. Am J Med Genet 1998; 78:140-45.

22. Tubbs RS, Oaks WJ. Chiari I malformation, caudal regression syndrome, and Pierre Robin syndrome: a previously unreported combination. 2006; 22:1507-8. 